\title{
Suppressing Riser-Based Slugging in Multiphase Flow by State Feedback
}

\author{
Hardy B. Siahaan, Ole M. Aamo, and Bjarne A. Foss
}

\begin{abstract}
This paper proposes a state feedback design method for attenuating severe slugging in multiphase flow pipeline systems. The feedback is designed based on the inputoutput linearization method, and incorporates the saturation effect on the input. The designed feedback can suppress the slugging phenomena provided some sufficient conditions are satisfied. Finally, checking the conditions lead to the selection of the variable which is 'more relevant' to be controlled.
\end{abstract}

\section{INTRODUCTION}

The theoretical development of stabilisation of multiphase flow in oil-production pipelines is still in its infancy. The stabilisation is related to the purpose of suppressing an oscillation phenomenon, called severe slugging, that occurs in pipelines carrying multiphase flow. Severe slugging in pipelines is caused by inclined or vertical pipe sections, and is potentially damaging to downstream processing equipment such as separators. Moreover, large oscillations may cause lower oil production. While the traditional remedy is to manually choke the flow at the expense of lower production, automatic control has the potential of removing oscillations without production loss. It is therefore essential to develop control strategies that guarantee attenuation of severe slugging.

An important step in the development of a stabilisation scheme in this direction can be traced back to [6], where it was shown that active choking could remove oscillations in a vertical riser. In [3], [4], [5], it was shown that by stabilising the riser base pressure by active choking, large oscillations are effectively removed. Despite the fact that active control manages to suppress slugging, none of the previous works, to the best of our knowledge, has proved from a mathematical point of view why the control scheme works.

In this paper, we design a state feedback control law which is able to suppress severe slugging occurring in the model developed in [8]. Theoretically, the feedback can achieves regulation of the output to its set-point. The feedback is based on the input-output linearization approach, where the output is chosen such that it satisfies certain conditions.

\section{MODEL}

Mathematical models of multiphase flow can be found in for instance [1], [2], [8], [9], and are usually of different type depending on the application and the assumptions made. In this paper, we consider a mathematical model of

This work was supported by the Gas Technology Center at NTNU, Statoil and the Norwegian Research Council

All authors are with the Department of Engineering Cybernetics, Norwegian University of Science and Technology, 7491 Trondheim, Norway.

E-mail of corresponding author: siahaan@itk.ntnu.no

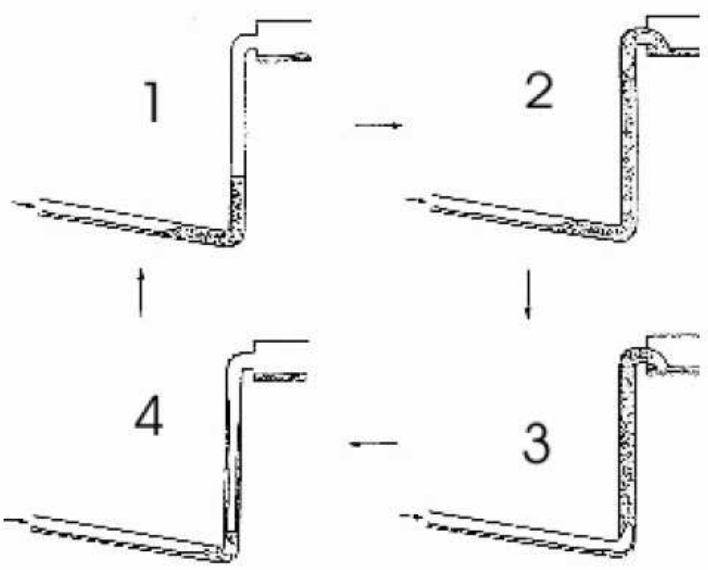

Fig. 1. Severe slugging in the pipeline-riser system

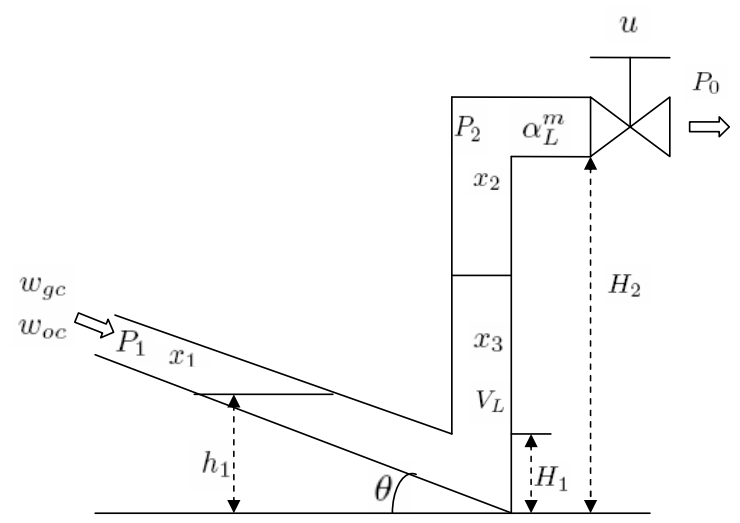

Fig. 2. The pipeline-riser system with parameters

multiphase flow [8] which captures gravity-induced slugging in a pipeline-riser system where the inclination of the pipe may vary from case to case, while the riser is vertical. Generally, severe slugging in the riser can be described as follows (see Fig. 1). When multiphase flow (gas and liquid) enter the riser at relatively low rate, the liquid stays in the riser base. The liquid will block the gas from entering the riser until the pressure of the gas upstream the riser base can overcome the hydrostatic pressure of the liquid in the riser. When the pressure of the gas is high enough, the gas penetrates into the riser, violently pushing the accumulated liquid out of the riser. This behaviour causes high fluctuations in the separator, and may damage it. 
The model (see Fig. 2) can be written as

$$
\begin{aligned}
& \dot{x}_{1}=w_{g c}-w_{g}(x), \\
& \dot{x}_{2}=w_{g}(x)-w_{g p}(x, u), \\
& \dot{x}_{3}=w_{o c}-w_{o p}(x, u),
\end{aligned}
$$

where $x=\left[x_{1}, x_{2}, x_{3}\right]^{T}$ is the state of the system, $x_{1}$ is the total mass of gas in the volume upstream of the riser base (volume one), $x_{2}$ is the total mass of gas in the riser (volume two), $x_{3}$ is the total mass of oil, $u$ is the opening position of the production orifice (control input to the system), $w_{g c}$ is the constant mass flow rate of gas into volume one, $w_{g}$ is the mass flow rate of gas from volume one into volume two, $w_{g p}$ is the mass flow rate of gas through the production orifice, $w_{o c}$ is the constant mass flow rate of oil entering the riser, and $w_{o p}$ is the mass flow rate of produced oil through the production orifice. The non-constant flows in equation (1)-(3) are expressed as

$$
\begin{aligned}
w_{g}(x) & =v_{G 1}(x) \rho_{G 1}\left(x_{1}\right) \widehat{A}(x), \\
w_{g p}(x, u) & =\left(1-\alpha_{L}^{m}(x)\right) w_{p}(x) u, \\
w_{o p}(x, u) & =\alpha_{L}^{m}(x) w_{p}(x) u,
\end{aligned}
$$

where $v_{G 1}$ is the gas velocity at the riser base, $\rho_{G 1}$ is the density of gas in volume one, $\widehat{A}$ is the gas flow area at the riser base, $\alpha_{L}^{m}$ is the oil fraction (mass basis) through the valve, and $w_{p}$ is the total mass flow rate through the valve when it is fully open. They are given by

$$
\begin{aligned}
v_{G 1}(x) & =\left\{\begin{array}{c}
K_{2} \frac{H_{1}-h_{1}(x)}{H_{1}} \sqrt{\frac{P_{1}\left(x_{1}\right)-P_{2}(x)-\rho_{L} g H_{2} \alpha_{L}(x)}{\rho_{G 1}\left(x_{1}\right)},} \\
\text { if } h_{1}(x)<H_{1} \\
\text { otherwise }
\end{array}\right. \\
\rho_{G 1}\left(x_{1}\right) & =\frac{x_{1}}{V_{G 1}}, \\
\alpha_{L}^{m}(x) & =\alpha_{L T}(x) \frac{\rho_{L}}{\rho_{T}(x)}, \\
\widehat{A}(x) & =r^{2}[\pi-\varphi(x)-\cos (\pi-\varphi(x)) \sin (\pi-\varphi(x))], \\
w_{p}(x) & =K_{1} \sqrt{\rho_{T}(x)\left[P_{2}(x)-P_{0}\right]},
\end{aligned}
$$

where $K_{2}$ is the internal gas flow constant, $H_{1}$ is the critical oil level, $H_{2}$ is the height of the riser, $\rho_{L}$ is the density of oil, $g$ is the specific gravity, $V_{G 1}$ is the size of volume one, $r$ is the radius of the pipe, $K_{1}$ is the valve constant, and $P_{0}$ is the constant pressure after the valve. The liquid level at the riser base $\left(h_{1}\right)$, the pressure in volume one $\left(P_{1}\right)$, the pressure in volume two $\left(P_{2}\right)$, the average liquid fraction (volume basis) in the riser $\left(\alpha_{L}\right)$, the angle $\varphi$, the liquid fraction (volume basis) through the valve $\left(\alpha_{L T}\right)$ and the density through the valve $\left(\rho_{T}\right)$ are given by

$$
\begin{aligned}
h_{1}(x) & =\frac{V_{L}\left(x_{3}\right)-V_{L R}(x)}{A_{1}}, \\
P_{1}\left(x_{1}\right) & =\frac{x_{1} R T}{M_{G} V_{G 1}}, \\
P_{2}(x) & =\frac{x_{2} R T}{M_{G} V_{G 2}(x)}, \\
\alpha_{L}(x) & =\frac{V_{L R}(x)}{V_{T}}, \\
\varphi(x) & =\cos ^{-1}\left(\frac{\left(H_{1}-h_{1}\right) \cos \theta}{r}-1\right), \\
\alpha_{L T}(x) & =\left\{\begin{array}{c}
\frac{V_{L R}(x)-A_{2} H_{2}}{A_{3} H_{3}(1+w(x))}+\frac{w(x)}{1+w(x)} \alpha_{L}, \\
\text { if } V_{L R}(x)>A_{2} H_{2} \\
1+w(x)
\end{array},\right. \\
\rho_{T}(x) & =\alpha_{L T}(x) \rho_{L}+\left(1-\alpha_{L T}(x)\right) \rho_{G 2}(x),
\end{aligned}
$$

where $A_{1}$ is the cross section area in the horizontal plane upstream the riser base, $R$ is the gas constant, $T$ is the constant system temperature, $M_{G}$ is the molecular weight of gas, $V_{T}$ is the total volume of the riser, $\theta$ is the inclination of the feed pipe, $A_{2}$ is the cross sectional area in the horizontal plane of the riser, $A_{3}$ is the cross sectional area of the horizontal top section and $H_{3}$ is the length of the horizontal top section. The volume occupied by the oil $\left(V_{L}\right)$, the volume of the oil in the riser $\left(V_{L R}\right)$, the size of volume two $\left(V_{G 2}\right)$, the friction function $(w)$ and the gas density in volume two $\left(\rho_{G 2}\right)$ are given by

$$
\begin{aligned}
V_{L}\left(x_{3}\right) & =\frac{x_{3}}{\rho_{L}}, \\
V_{G 2}(x) & =V_{T}-V_{L R}(x), \\
V_{L R}(x) & =\frac{\rho_{\text {mix }}(x) V_{T}-x_{2}}{\rho_{L}}, \\
w(x) & =\frac{K_{3} \rho_{G 1}\left(x_{1}\right) v_{G 1}^{2}(x)}{\left(\rho_{L}-\rho_{G 1}\left(x_{1}\right)\right)^{n}}, \\
\rho_{G 2}(x) & =\frac{x_{2}}{V_{G 2}(x)},
\end{aligned}
$$

where $\rho_{L}$ is the liquid density, $n$ is the tuning parameter in the friction expression, and $K_{3}$ is the friction parameter. The average density in the riser $\left(\rho_{m i x}\right)$ satisfies the relation

$$
\begin{gathered}
\rho_{\text {mix }}(x) g\left(H_{2}+H_{3}\right)-\rho_{L} g h_{1}(x)=P_{1}\left(x_{1}\right)-P_{2}(x) . \\
\text { III. StATE FEEDBACK DESIGN BASED ON INPUT } \\
\text { OUTPUT LINEARIZATION }
\end{gathered}
$$

\section{State FeEdBack Design BASEd on INPUT OUTPUT LINEARIZATION}

In this section we design a state feedback control law which is based on the input-output linearization technique [7]. The output in this case is a variable which is chosen by the designer and it has to satisfy certain conditions.

\section{A. Feedback Design}

Suppose we select $\psi(x)$ and $u$ as the variable to be controlled (the output) and the manipulated variable (the input), respectively. The selected variable $\psi(x)$ should be continuous and bounded on a domain $D$. Throughout the 
paper we assume that the set $\Omega_{D}:=\left\{\psi(x) \in \mathbb{R}_{+} \mid x \in D\right\}$ contains all admissible $\psi$.

We can rewrite the system as

$$
\begin{aligned}
& \dot{x}_{1}=w_{g c}-w_{g}(x, \psi) \\
& \dot{x}_{2}=w_{g}(x, \psi)-\left[1-\alpha_{L}^{m}(x, \psi)\right] w_{p}(x, \psi) u \\
& \dot{x}_{3}=w_{o c}-\alpha_{L}^{m}(x, \psi) w_{p}(x, \psi) u
\end{aligned}
$$

and the dynamics of the variable to be controlled can be expressed as

$$
\dot{\psi}=f_{\psi}(x)+g_{\psi}(x) u
$$

where

$$
\begin{aligned}
f_{\psi}(x)= & \frac{\partial \psi}{\partial x_{1}}\left(w_{g c}-w_{g}(x, \psi)\right)+\frac{\partial \psi}{\partial x_{2}} w_{g}(x, \psi) \\
& +\frac{\partial \psi}{\partial x_{3}} w_{o c}, \\
g_{\psi}(x)= & -\left[\frac{\partial \psi}{\partial x_{2}}\left(1-\alpha_{L}^{m}(x, \psi)\right)+\frac{\partial \psi}{\partial x_{3}} \alpha_{L}^{m}(x, \psi)\right] \times \\
& w_{p}(x, \psi) .
\end{aligned}
$$

We assume that the selection of $\psi(x)$ guarantees that the following assumptions are satisfied.

Assumption $1 g_{\psi}(x) \neq 0$ for $x \in D$.

Assumption 2 The sets

$$
\begin{aligned}
& \left\{x \in D \mid g_{\psi}(x)<0, f_{\psi}(x) \leq 0, \psi(x)-\psi^{*} \leq 0\right\} \\
& \left\{x \in D \mid g_{\psi}(x)>0, f_{\psi}(x) \geq 0, \psi(x)-\psi^{*} \geq 0\right\}
\end{aligned}
$$

are empty.

Assumption $3\left|g_{\psi}(x)\right| \geq\left|f_{\psi}(x)\right|$ for $x \in D$.

Proposition 1 Under Assumption 1 and the feedback

$$
u=\frac{f_{\psi}(x)+\lambda\left(\psi(x)-\psi^{*}\right)}{-g_{\psi}(x)}
$$

where $\lambda>0$, the equilibrium point $\psi=\psi^{*}$ is asymptotically stable.

Proof: By Assumption 1 the feedback (13) does not have any singularity in the domain $D$. Applying the feedback scheme (13) in (10) yields

$$
\dot{\psi}=-\lambda\left(\psi-\psi^{*}\right)
$$

Consider the Lyapunov function candidate $V=\left(\psi-\psi^{*}\right)^{2}$. Then its time derivative

$$
\dot{V}=-2 \lambda\left(\psi-\psi^{*}\right)^{2}
$$

is negative definite.

In applications, the feedback in the form (13) has to be saturated since $u$ is the valve opening which is in the range between zero and one. The following theorem presents the result on saturated feedback.
Theorem 1 Consider

$$
\begin{aligned}
\dot{\psi} & =f_{\psi}(x)+g_{\psi}(x) \tilde{u} \\
\tilde{u} & := \begin{cases}0, & \text { if } u<0 \\
u, & \text { if } 0 \leq u \leq 1 \\
1, & \text { if } u>1\end{cases}
\end{aligned}
$$

Under Assumption 1, 2 and 3 and the feedback $u$ in the form (13), the equilibrium point $\psi=\psi^{*}$ is asymptotically stable.

Proof: Consider the Lyapunov function candidate $V=$ $\left(\psi-\psi^{*}\right)^{2}$. We have $\dot{V}=2\left(\psi-\psi^{*}\right) \dot{\psi}$.

1) Case $0 \leq u \leq 1$ : See the proof of Proposition 1 .

2) Case $u<0$ : If $g_{\psi}(x)<0$, then (13) gives $f_{\psi}(x)<$ $\lambda\left(\psi^{*}-\psi(x)\right)$. Then by (11) of Assumption 2 we have

$$
\dot{V}=2\left(\psi-\psi^{*}\right) f_{\psi}(x)<0 .
$$

The result in the case of $g_{\psi}(x)>0$ is achieved similarly using (12).

3) Case $u>1$ : For the case $g_{\psi}(x)<0$, (13) implies $f_{\psi}(x)+g_{\psi}(x)>\lambda\left(\psi^{*}-\psi(x)\right)$. By Assumption 3 we then have $0>f_{\psi}(x)+g_{\psi}(x)>\lambda\left(\psi^{*}-\psi(x)\right)$ which implies $\psi(x)-\psi^{*}>0$. Then

$$
\dot{V}=2\left(\psi-\psi^{*}\right)\left(f_{\psi}(x)+g_{\psi}(x)\right)<0 .
$$

The result in the case of $g_{\psi}(x)>0$ is achieved similarly.

Remark 1 Assumption 1 is imposed to avoid singularity in the feedback (13).

Remark 2 Assumption 2 is imposed for the case of saturation whenever the feedback (13) has negative value.

Remark 3 In Subsection III-B we use another condition to replace Assumption 1 and 2. It is sufficient to have

$$
f_{\psi}(x) g_{\psi}(x)<0 \text { for } x \in D
$$

to guarantee Assumption 1 and 2 to hold. The main reason for this is that, based on exhaustive simulation runs, the new condition is also necessary for Assumption 1 and 2.

Remark 4 Assumption 3 is imposed when $u>1$ since we need to show that $\left[f_{\psi}(x)+g_{\psi}(x)\right] g_{\psi}(x)>0$ in the proof of Theorem 1. However, the saturated feedback scheme may still guarantee convergence when $\left|g_{\psi}(x)\right|<\left|f_{\psi}(x)\right|$. For example, in the case $g_{\psi}(x)<0$ where $f_{\psi}(x)+g_{\psi}(x)>0$ at some $x$ it follows that $f_{\psi}(x)+g_{\psi}(x)>\max \left[0, \lambda\left(\psi^{*}-\psi(x)\right)\right]$ which gives

$$
\dot{V}=2\left(\psi-\psi^{*}\right) \dot{\psi}=2\left(\psi-\psi^{*}\right)\left(f_{\psi}(x)+g_{\psi}(x)\right)
$$

and thus

$$
\begin{array}{r}
\dot{V}>0, \quad \text { if } \psi-\psi^{*}>0 \\
\dot{V}<-2\left(\psi-\psi^{*}\right)^{2}, \quad \text { if } \psi-\psi^{*}<0 .
\end{array}
$$


Consequently convergence is guaranteed whenever $\psi-\psi^{*}<$ 0 . In the case of $\psi-\psi^{*}>0$, no conclusion can be made.

In practical applications, the model of multiphase flow in Section II may be modified to meet certain objectives. For instance, the equation for the production valve may vary depending on the type of valve being used. In the case of modification of the terms $w_{g}, w_{g p}, w_{o p}$ in the model, the feedback (13) can easily be modified. Thus, our feedback is quite flexible to modification of the model.

\section{B. Selection of Variable-To-Be-Controlled}

This subsection provides some approaches on selecting the controlled variables $\psi$ which fit the proposed feedback scheme. These approaches are not exact, but to some extent they can be used as guidelines for selecting the controlled variables. In short the approaches should guarantee that Assumption 1,2 and 3 are satisfied.

1) Empirical Approach: The first approach is to plot the functions $f_{\psi}$ and $g_{\psi}$ for a given data set and then select the variables, which satisfy Assumption 1 and 2, from the plots. For this purpose we run a simulation of the system with a given set of parameters. In this case the parameters are set so that

$$
\begin{array}{ll}
V_{G 1}=12.158 \mathrm{~m}^{3} & \rho_{L}=750 \mathrm{~kg} / \mathrm{m}^{3} \\
\theta=0.0274 \mathrm{rad} & H_{1}=0.12 \mathrm{~m} \\
H_{2}=300 \mathrm{~m} & H_{3}=0.12 \mathrm{~m} \\
L_{3}=100 \mathrm{~m} & A_{1}=0.4128 \mathrm{~m}^{2} \\
A_{2}=0.0113 \mathrm{~m}^{2} & A_{3}=12 \mathrm{~m}^{2} \\
R=8314 \mathrm{~J} /(\mathrm{K} * \mathrm{Kmol}) & T=308 \mathrm{~K} \\
M_{G}=35 \mathrm{~kg} / \mathrm{Kmol} & g=9.81 \mathrm{~m} / \mathrm{s}^{2} \\
P_{0}=50 \times 10^{5} \mathrm{~N} / \mathrm{m}^{2} & V_{T}=4.8329 \mathrm{~m}^{3} \\
n=2.55 & K_{1}=0.0051 \\
K_{2}=4.3983 & K_{3}=0.2030
\end{array}
$$

The opening of the production orifice is set to $50 \%(u=0.5)$. The simulation is run for 30 minutes and the states oscillate as shown in Fig. 3. This means that the constant input of 50\% opening induces severe slugging. Note that our purpose in the end is to stabilise a variable at a certain set-point and then to see whether it will suppress the slugging or not. A set-point here means a point which is associated with the equilibrium condition $\dot{x}=0$, when a certain constant input $u$ is applied. In this case the input $u$ could be a constant value between zero and one.

The candidates for the controlled variable are $\rho_{G 1}, P_{1}, V_{L}$, $\rho_{m i x}, V_{L R}, h_{1}, V_{G 2}, P_{2}, \alpha_{L}, \phi, A, v_{G 1}, \rho_{G 2}, w, \alpha_{L T}, \rho_{T}$, $\alpha_{L}^{m}$ and $w_{g}$. All these variables are dependent on the state $x$ (see Section II). Based on the available data set for 30 minutes the only variables which satisfy (16) are $V_{L}, h_{1}, P_{2}$ and $\rho_{G 2}$ (see Fig. 4). Note that we skip the plots associated with $\rho_{G 2}$ as they are equivalent to those with $P_{2}$ (see also from the equations of $\rho_{G 2}$ and $P_{2}$ in Section II). The next step is to check whether Assumption 3 is also satisfied for $V_{L}, h_{1}$ and $P_{2}$. Fig. 4 shows that none of the chosen variables satisfy the required condition of Assumption 3.

We should keep in mind that the current selection process is based on the data set of severe slugging where large
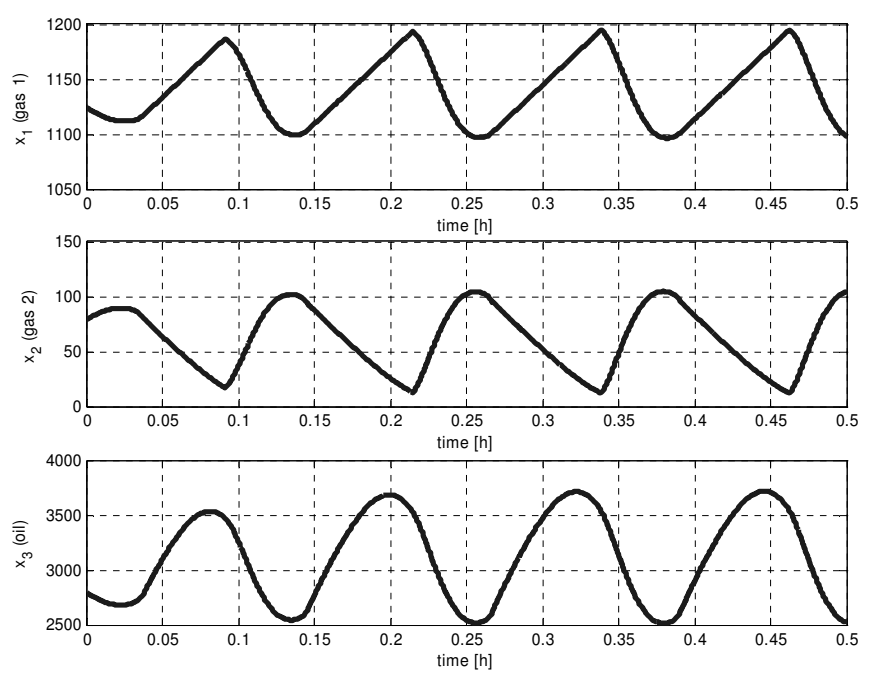

Fig. 3. The state $x$ for $u=0.5$
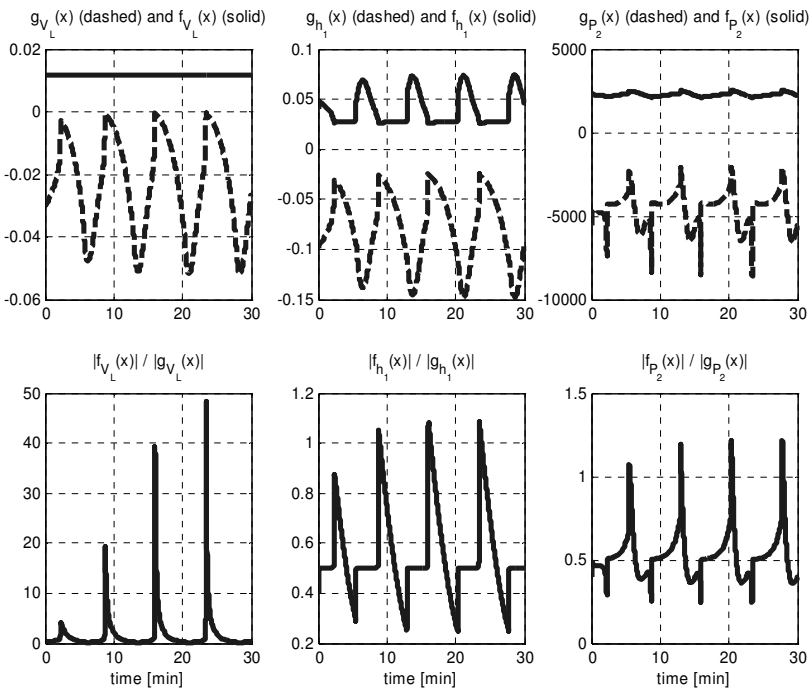

Fig. 4. $g_{\psi}(x), f_{\psi}(x)$ and $\left|f_{\psi}(x)\right| /\left|g_{\psi}(x)\right|$ for $V_{L}, h_{1}$ and $P_{2}$ in the case $u=0.5$

magnitude of oscillatory behavior of the state $x$ is expected. This is the reason why at some times Assumption 3 is not satisfied for $V_{L}, h_{1}$ and $P_{2}$. As a comparison we perform another simulation for $10 \%$ of opening of the production orifice which is in the stable region. The response of the state to the input $u=0.1$, when the initial condition is associated with an oscillatory behavior, can be seen from Fig. 5 where the state finally converges to a set-point after oscillating. Fig. 6 shows the corresponding plots of $g_{\psi}(x)$, $f_{\psi}(x)$ and $\left|f_{\psi}(x)\right| /\left|g_{\psi}(x)\right|$ for $V_{L}, h_{1}$ and $P_{2}$. It indicates that during the small magnitude of oscillation of the state $x$, the magnitude of function $f_{\psi}$ is always smaller than that of $g_{\psi}$. Thus we can say that whenever the magnitude of oscillation is small we can select $V_{L}, h_{1}$ and $P_{2}$ as the controlled variable in our scheme.

2) Analytical and Empirical Approach: The next approach is a mixture of analytical and empirical nature. The 

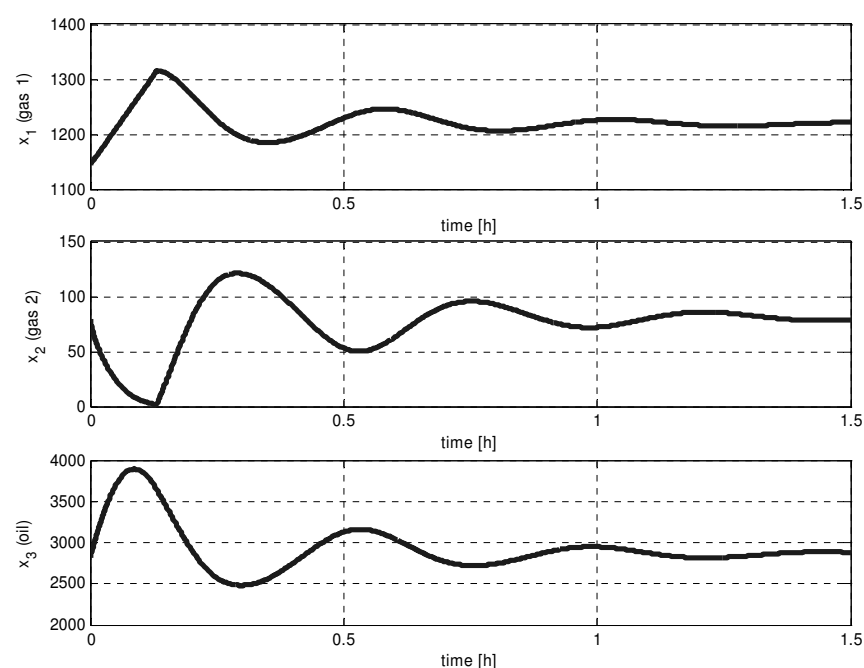

Fig. 5. The state $x$ for $u=0.1$
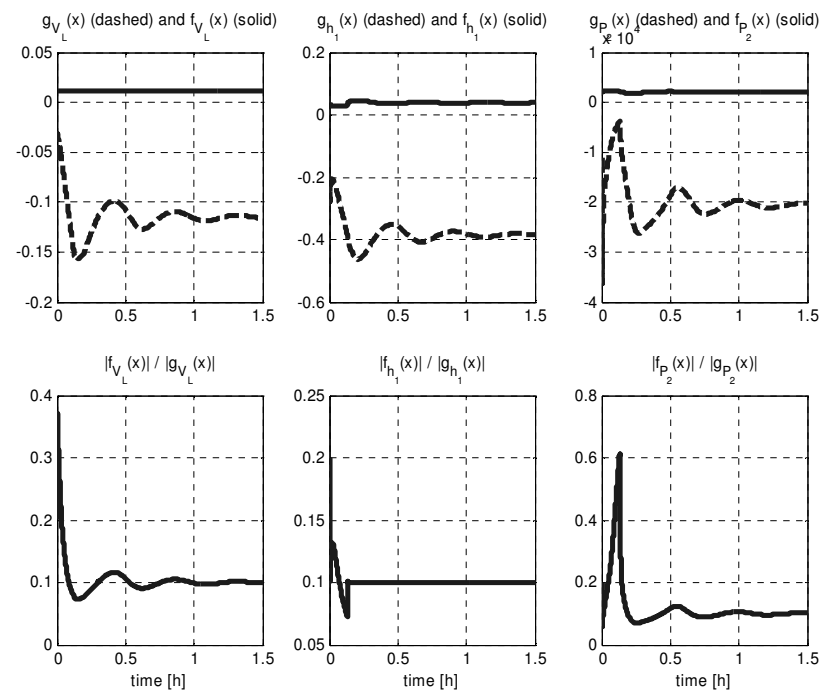

Fig. 6. $g_{\psi}(x), f_{\psi}(x)$ and $\left|f_{\psi}(x)\right| /\left|g_{\psi}(x)\right|$ for $V_{L}, h_{1}$ and $P_{2}$ in the case $u=0.1$

candidates to be assessed by this approach is the total mass of the liquid $x_{3}$ and the total mass $M=x_{1}+x_{2}+x_{3}$. We then obtain

$$
\begin{aligned}
f_{x_{3}}(x)=w_{o c}>0, & g_{x_{3}}(x)=-\alpha_{L}^{m}(x) w_{p}(x)<0 \\
f_{M}(x)=w_{g c}+w_{o c}>0, & g_{M}(x)=-w_{p}(x)<0
\end{aligned}
$$

where $f_{\psi}(x)$ is a constant for both cases. By (16), Assumption 1 and 2 are satisfied. In the event of severe slugging, as a result of blocking, the total mass flow rate through the production valve $\left(w_{p}(x)\right)$ and the oil fraction (mass basis) through the production valve $\left(\alpha_{L}^{m}(x)\right)$ become very small. As a result the absolute value of $g_{\psi}(x)$ is very small compared to that of $f_{\psi}(x)$ for $x_{3}$ and $M$. Consequently Assumption 3 is not satisfied. On the other hand, when there is no slugging, using the same reasoning we can guarantee that the absolute value of $g_{\psi}(x)$ is big enough to satisfy Assumption 3 for $x_{3}$ and $M$.
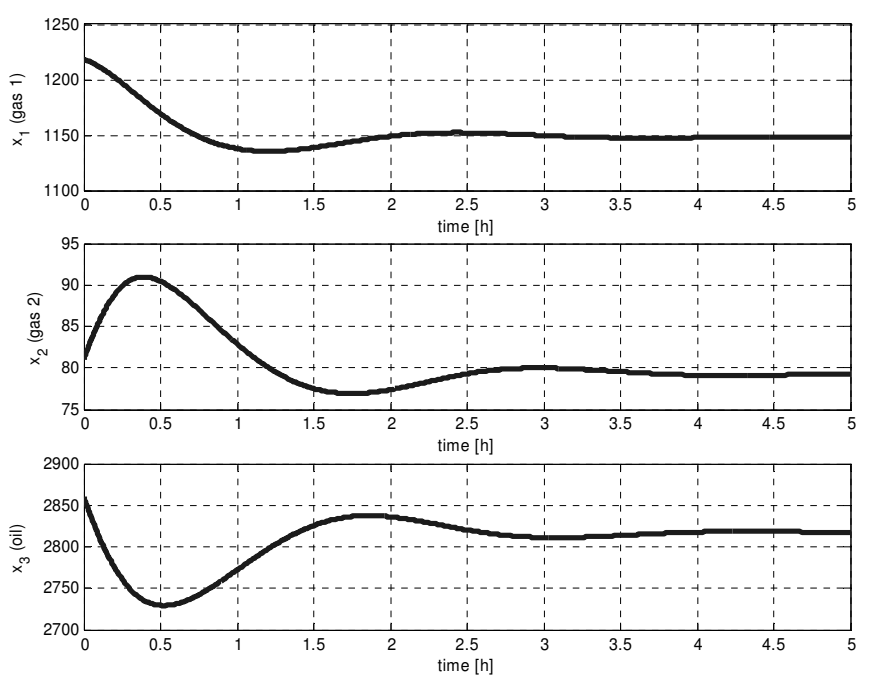

Fig. 7. The state $x$ (when $h_{1}$ is the controlled variable)
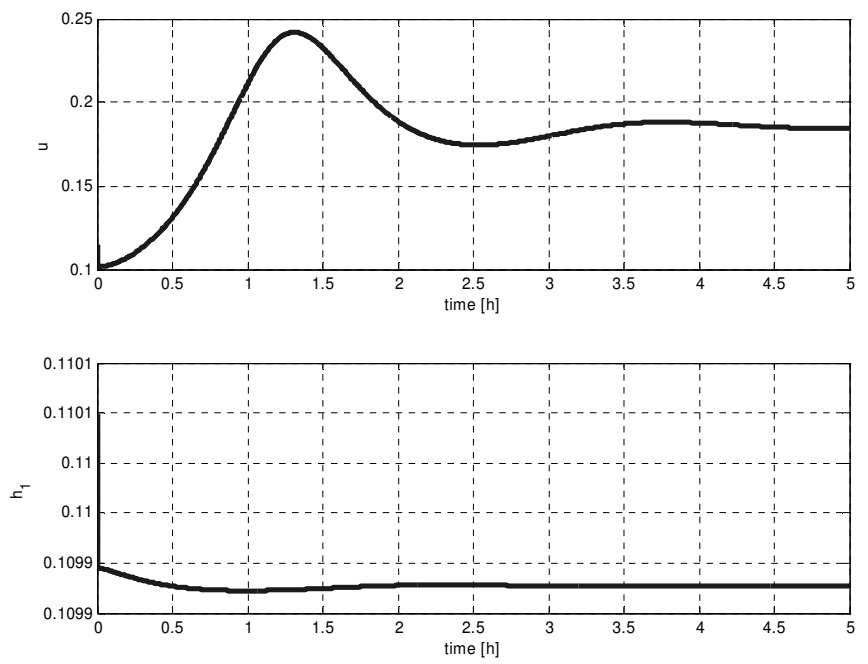

Fig. 8. The feedback $u$ and the liquid level $h_{1}$ as the controlled variable

3) Closed Loop Investigation: In real applications, especially for safety reasons, large oscillatory behavior is avoided. The large magnitude of oscillation can be avoided during stabilisation of an unstable set-point by selecting an initial set-point which is associated with the stable region (the opening of the production orifice which does not induce slugging). By slowly changing the set-point from the stable one to the slugging region while the controller is working, stabilisation is also achieved.

For closed loop simulation with the feedback when the controlled variable is $h_{1}$, we set $\lambda=2 \times 10^{8}$. The initial condition is set to the point associated with the stable region of constant opening $u=0.1$. The purpose of the feedback is to stabilise $h_{1}$ at the point 0.1099 which is associated with the equilibrium condition of $u=0.2$ (slugging case). In Fig. 7 we can see that the states converge to a point and the state feedback does not experience saturation (Fig. 8). Unfortunately the state feedback $u$ does not converge to 

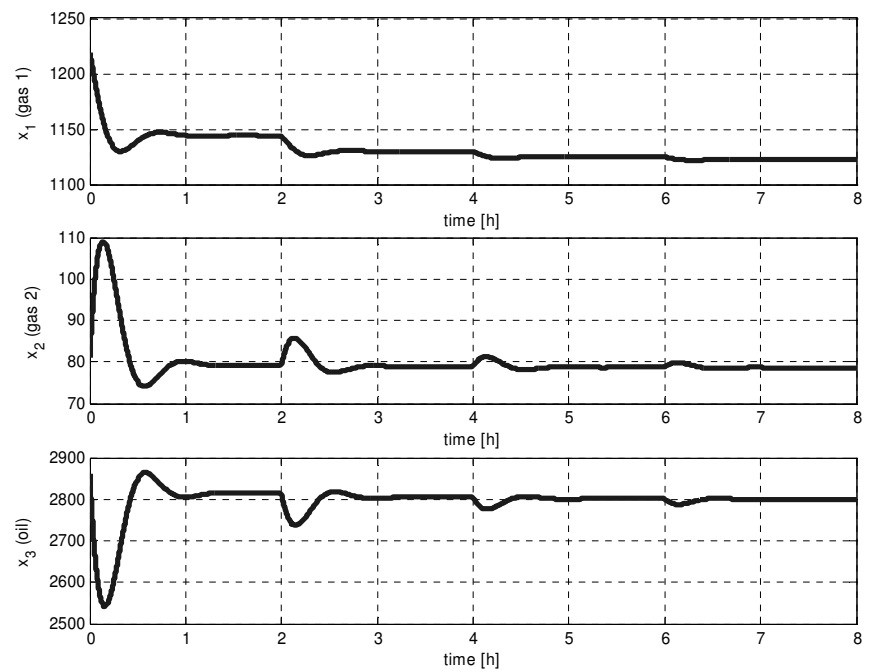

Fig. 9. The state $x$ (when $P_{2}$ is the controlled variable)

the desired set point of 0.2 . Instead the state feedback $u$ converges to the point 0.1877 . To understand what happen we can observe the plot of $h_{1}$ in Fig. 8. The variable $h_{1}$ converges to the point 0.1099 which is the point associated with the equilibrium condition of $u=0.2$. But apparently the point $h_{1}=0.1099$ is also associated with the equilibrium condition of $u=0.1877$. In this case the controller actually works well that the controlled variable $h_{1}$ converges to 0.1099 even though $u$ converges to another point. In this case the internal dynamic of the system does not evolve as what is expected. Thus the selection of $h_{1}$ as the variable to be controlled has a drawback in that the state $x$ is 'unobservable' from the information given by $h_{1}$.

With the same initial condition we try another feedback where the variable to be controlled is $P_{2}$. The constant $\lambda$ is set to 3000 . The feedback is designed in such a way that the system moves from the initial condition associated with the equilibrium condition of $u=0.1$, gradually with step 0.1 , to the final one of $u=0.5$. The results of the simulation using the feedback can be seen in Fig. 9 and Fig. 10 where all the states are converging and the feedback also converges to the desired points. Thus the feedback works well when the variable to be controlled is $P_{2}$. The same type of results are also obtained when we select $M$ and $x_{3}$ (equivalently $V_{L}$; see Section II) as the controlled variable.

Clearly, the foregoing stabilisation scenario of $P_{2}, x_{3}$ and $M$ indicates that severe slugging can be suppressed as satisfactorily demonstrated by the results of simulation of the states. Validating the results analytically needs a further investigation which is not easy since the model is quite complicated. However, validation can be done easily in the case of selecting $x_{3}$ as the controlled variable. Stabilising the controlled variable $x_{3}$ at a set-point guarantees that the total mass of liquid (oil) does not fluctuate and thus no blockage occurs. In this case severe slugging can be avoided and we can ignore whether $x_{1}$ and $x_{2}$ oscillate or not.
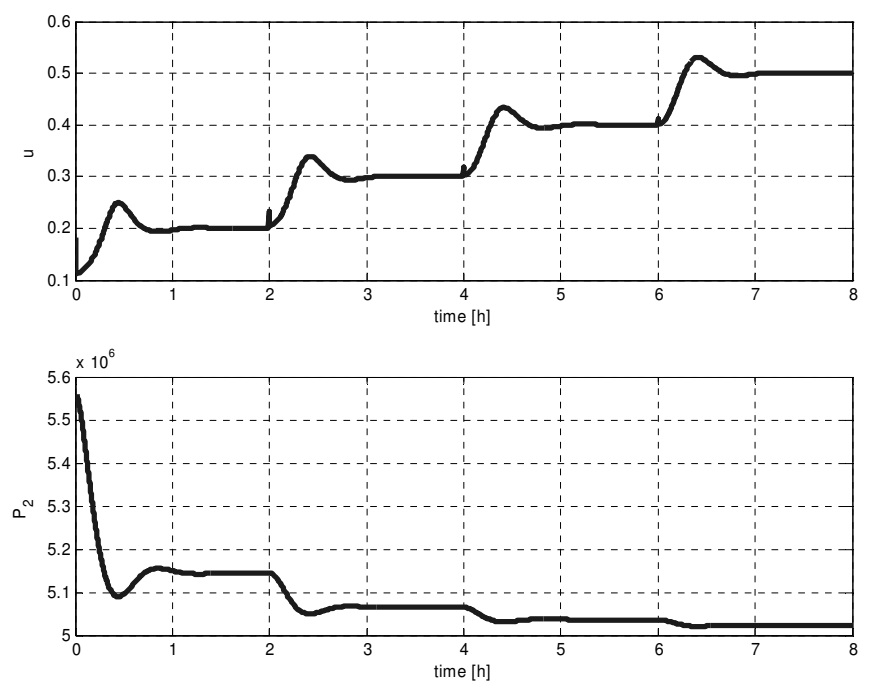

Fig. 10. The feedback $u$ and the pressure $P_{2}$ as the controlled variable

\section{CONCLUDING REMARK}

An early phase in the design method of state feedback for the purpose of suppressing riser-induced slugging occurring in a vertical pipeline has been presented. By carefully selecting the variable to be controlled the saturated feedback can guarantee asymptotic stability of the system and thus attenuate severe slugging.

\section{REFERENCES}

[1] O.M. Aamo, G.O. Eikrem, H.B. Siahaan and B.A. Foss, "Observer Design for Multiphase Flow in Vertical Pipes with Gas-Lift: Theory and Experiments", Journal of Process Control, 5, Issue 3, pp. 247-257, 2005.

[2] K. Bendiksen, D. Malnes and O. Nydal, "On the Modelling of Slug Flow", Chemical Engineering Science, 40, pp. 59-75, 1985.

[3] M. Dalsmo, E. Halvorsen and O. Slupphaug, "Active Feedback Control of Unstable Wells at the Brage Field", SPE Production and Facilities, SPE 77650, 2002.

[4] K. Havre and M. Dalsmo, "Active Feedback Control as a Solution to Severe Slugging", SPE Production and Facilities, SPE 79252, pp. 138-148, 2002.

[5] K. Havre, K.O. Stornes and H. Stray, 'Taming Slug Flow in Pipelines", ABB Review, 4, Dec. 2000.

[6] P. Hedne and H. Linga, "Supression on Terrain Slugging with Automation and Manual Riser Choking", Advances in Gas-Liquid Flows, pp. 453-460, 1990.

[7] H. K. Khalil. Nonlinear Systems, Prentice Hall, 3rd. ed. 2002.

[8] E. Storkaas, S. Skogestad and J. Godhavn, "A Low Dimentional Model of Severe Slugging for Controller Design and Analysis", Proc. Multi Phase '03, San Remo, Italy, 11-13 June 2003.

[9] Y. Taitel and D. Barnea, "Two Phase Slug Flow", Advances in Heat Transfer, 20, pp. 71-103, 1990. 